\title{
Doença de Crohn: aspectos do tratamento na atualidade
}

\section{Crohn's disease: aspects of current treatment}

Gabriel Fernandes de Sousa ${ }^{1}$. Keli Camila Vidal Grochoski ${ }^{1}$. Rayanir de Freitas Marinho ${ }^{1}$. Matheus Fragoso Vieira $^{1}$. Rafael Gonzaga Nahoum ${ }^{1}$.

1 Faculdade de Ciências Médicas da Paraíba, João Pessoa, Paraíba, Brasil.

\section{RESUMO}

Objetivo: Desvelar a produção científica mais recente a respeito da terapêutica utilizada no tratamento da doença de Crohn (DC). Métodos: Trata-se de uma revisão integrativa da literatura em que se realizou a busca das publicações na Biblioteca Virtual em Saúde (BVS). Foi utilizada a base de dados MEDLINE, através de estudos disponíveis para visualização em revistas de gastroenterologia publicadas entre 2012 e 2016, totalizando 33 artigos. Resultados: Dentre os artigos analisados, 81,8\% abordaram a terapia farmacológica, que consiste principalmente em esteroides e imunossupressores e, nos pacientes não responsivos, compostos biológicos como anti-TNF alfa. $\mathrm{O}$ adalimumabe apresentou mais efeitos adversos do que o infliximabe. As tiopurinas têm sido associadas à acometimentos hepáticos. A nutrição parenteral total mostrou-se importante, sendo recomendada para pacientes com DC moderada a grave e a nutrição enteral exclusiva para tratamento de primeira linha em crianças com DC. Mostrou-se uma necessidade decrescente de cirurgia após o uso de imunomoduladores. O transplante de microbiota fecal foi relatado como uma terapia alternativa em casos de DC refratária complicada. Conclusões: Observou-se a prevalência de estudos acerca da terapia farmacológica e a necessidade da realização de novos estudos sobre as opções terapêuticas da DC, principalmente no que diz respeito à terapia não farmacológica.

Palavras-chave: Doença de Crohn. Tratamento. Gastroenterologia.

\section{ABSTRACT}

Objective: To uncover the most recent scientific production regarding the therapy used in the treatment of Crohn's disease (CD). Methods: This is an integrative review of the literature which the search was carried out in the publications of the Biblioteca Virtual de Saúde (BVS). The MEDLINE database was used through studies available for viewing in gastroenterology journals published between 2012 and 2016, totaling 33 articles. Results: Among the analyzed articles, 81,8\% addressed pharmacological therapy, which consists mainly of steroids and immunosuppressants and, in nonresponsive patients, biological compounds such as anti-TNF alpha. Adalimumab had more adverse effects than infliximab. Thiopurines have been associated with hepatic impairment. Total parenteral nutrition has shown to be important and is recommended for patients with moderate to severe CD and exclusive enteral nutrition for first-line treatment in children with $\mathrm{CD}$. There has been a decreasing need for surgery after the use of immunomodulators. Fecal microbiota transplantation has been reported as an alternative therapy in cases of complicated refractory CD. Conclusions: The prevalence of studies on pharmacological therapy and the need for new studies on the therapeutic options of $\mathrm{CD}$, especially with regard to non-pharmacological therapy, were observed.

Keywords: Crohn Disease. Therapeutics. Gastroenterology.

Autor correspondente: Gabriel Fernandes de Sousa, Faculdade de Ciências Médicas da Paraíba, BR 230 - Km 09, Intermares, Cabedelo, Paraíba, Brasil. CEP: 58106-402. Telefone: +55 83 3248-1035. E-mail: gabrielfernanddes@hotmail.com

Conflito de interesses: Não há qualquer conflito de interesses por parte de qualquer um dos autores.

Recebido em: 15 Ago 2018; Revisado em: 20 Mar 2019; Aceito em: 20 Mar 2019. 


\section{INTRODUÇÃO}

As doenças inflamatórias intestinais (DII), como a doença de Crohn (DC), são condições inflamatórias crônicas ${ }^{1-4}$ e recidivantes do intestino de etiologia desconhecida, ${ }^{1-3}$ associando-se a uma morbidade significativa. ${ }^{4}$ A DC ocorre principalmente em adultos, porém, $7 \%$ a $20 \%$ dos casos são diagnosticados na infância, sendo frequentemente descrita como mais grave e agressiva nas crianças do que nos adultos. ${ }^{5}$

$\mathrm{Na}$ DC, a inflamação pode envolver qualquer parte do trato digestivo, desde a cavidade oral até o ânus, sem solução de continuidade, podendo afetar todas as camadas da mucosa. ${ }^{2}$ A visão mais aceita sobre a fisiopatogenia da DII é que surge a partir de uma perda de tolerância a bactérias entéricas devido à genética e fatores ambientais, associadas a um comprometimento da função da barreira epitelial do intestino, ${ }^{1}$ ocorrendo em hospedeiros geneticamente susceptíveis mediante desencadeamento de influências ambientais. $^{2}$

As opções de tratamento para a DC têm se expandido ao longo dos últimos vinte anos com o surgimento dos anti fator de necrose tumoral alfa (anti-TNF- $\alpha$ ) e anticorpos anti-integrina. Entretanto, uma proporção significativa de pacientes com DC não responde aos medicamentos existentes. Há também uma necessidade não atendida de novos medicamentos para a doença de Crohn com mecanismos exclusivos de ação. Entretanto, com avanços significativos no entendimento da patogênese da DC, um número crescente de drogas candidatas tem surgido. ${ }^{3}$

As terapias biológicas com agentes que neutralizam o fator de necrose tumoral têm revolucionado o tratamento das doenças inflamatórias crônicas. No entanto, a capacidade de resposta é de difícil previsão, podendo haver diferenças na eficácia e modo de ação diferente entre os agentes. ${ }^{5}$ A cirurgia é frequentemente recomendada na falha do tratamento medicamentoso ou quando há retardo de crescimento em pacientes pediátricos com doença localizada. Historicamente, $50 \%$ a $90 \%$ de pacientes adultos com DC necessitam de ressecção do intestino em algum momento. ${ }^{6}$

Atualmente, temsurgidoumcrescentenúmerodemedicamentos disponíveis no mercado, constituindo uma ampla diversidade terapêutica. No entanto, foi observado que há uma escassez de estudos que reúnam os aspectos terapêuticos dessa patologia, como as interações, efeitos colaterais e contraindicações das drogas utilizadas na atualidade, bem como a uso da terapia não farmacológica, justificando-se a necessidade desse estudo. O presente estudo tem como objetivo desvelar a produção científica mais recente a respeito da terapêutica utilizada no tratamento da doença de Crohn.

\section{MÉTODOS}

Trata-se de uma revisão integrativa da literatura, a qual inclui a análise de pesquisas relevantes que dão suporte para a tomada de decisão e a melhoria da prática clínica. ${ }^{7}$ Esse método de pesquisa permite a síntese de múltiplos estudos publicados e possibilita conclusões gerais a respeito de uma particular área de estudo. ${ }^{8}$ Portanto, a revisão integrativa tem sido apontada como uma ferramenta ímpar no campo da saúde, pois sintetiza as pesquisas disponíveis sobre determinada temática e direciona a prática fundamentando-se em conhecimento científico. ${ }^{9}$

Para guiar esta pesquisa, formulou-se a questão sobre o que foi produzido na literatura a respeito do tratamento da Doença de Crohn. Realizou-se em setembro de 2016 a busca das publicações na Biblioteca Virtual em Saúde (BVS). Foi utilizado o cruzamento dos descritores "Crohn's disease OR Crohn"; "Crohn's disease AND Treatment"; "Crohn AND Treatment", sendo inicialmente encontrados 10.946 artigos.

Como critérios de inclusão foram selecionados os seguintes filtros: Texto completo disponível online; na Base de dados Medical Literature Analysis and Retrieval System Online (MEDLINE); cujo assunto principal fosse: Doença de Crohn, Anticorpos Monoclonais, Anti-Inflamatórios, Imunossupressores, Fator de Necrose Tumoral alfa, Fármacos Gastrointestinais, Anticorpos monoclonais Humanizados, Azatioprina, Anti-Inflamatórios não esteroides. De acordo com o tipo de estudo foram selecionados: Relato de Casos, Estudo de Coorte, Estudo de Casos e Controles; nos Limites: Humanos, Masculino, Feminino, Adulto, Meia-Idade, Adolescente, Idoso, Criança, Criança pré-escolar, Gravidez, Lactente, Recém-nascido; cujo idioma fosse Inglês e Português; cujo Assunto da Revista fosse Gastroenterologia; publicados nos anos de 2012 a 2016.

Após a avaliação dos resumos, foram selecionadas para o estudo as pesquisas disponíveis para a visualização que abordassem exclusivamente o tratamento da doença de Crohn, totalizando 33 artigos.

\section{RESULTADOS}

Ao final da análise, resultou um total de 33 artigos que se enquadravam aos critérios de inclusão, os quais estão apresentados no Quadro 1, sendo 20 estudos de coorte $(60,6 \%), 10$ relatos de caso $(30,3 \%), 02$ revisões sistemáticas associadas a metanálise $(6 \%)$ e 01 relato de caso e revisão de literatura (3\%). Tendo sido separados os eixos temáticos em dois grandes grupos: Terapia Farmacológica e Terapia Não Farmacológica da DC, na qual foram analisadas as questões a respeito desses grupos, sendo visto que não há uma terapia ao mesmo tempo adequada e segura para os pacientes.

Dos 33 artigos analisados, 12 foram do ano de $2012(36,4 \%)$, 08 de 2013 (24,2\%), 08 de 2014 (24,2\%), 03 de 2015 (9,1\%) e 02 de $2016(6,1 \%)$, sendo percebida uma carência de artigos acerca do tema nos anos de 2015 e 2016, que juntos somaram apenas 15,2\% dos artigos. Os tipos de estudo mais frequentes foram Estudo de Coorte e Relato de Caso, que somaram $90,9 \%$. Dentre os artigos, 27 tiveram como enfoque principal a terapia farmacológica $(81,8 \%)$ e apenas 06 deles abordaram a não farmacológica $(18,2 \%)$. 
Quadro 1. Descrição dos estudos incluídos na revisão integrativa, segundo título, tipo de estudo, periódicos, enfoque e ano de publicação.

\begin{tabular}{|c|c|c|c|c|}
\hline Título & Tipo de estudo & Periódico & Enfoque & Ano \\
\hline $\begin{array}{l}\text { A case of opportunistic skin infection with Mycobacterium } \\
\text { marinum during adalimumab treatment in a patient with } \\
\text { Crohn's disease. }\end{array}$ & Relato de Caso & J Crohns Colitis & $\begin{array}{l}\text { Terapia } \\
\text { Farmacológica }\end{array}$ & 2013 \\
\hline $\begin{array}{l}\text { A prospective cohort study to determine the relationship } \\
\text { between serum infliximab concentration and efficacy in } \\
\text { patients with luminal Crohn's disease. }\end{array}$ & Estudo de Coorte & $\begin{array}{l}\text { Aliment } \\
\text { Pharmacol Ther }\end{array}$ & $\begin{array}{l}\text { Terapia } \\
\text { Farmacológica }\end{array}$ & 2014 \\
\hline $\begin{array}{l}\text { A retrospective study showing maintenance treatment } \\
\text { options for pediatric } \mathrm{CD} \text { in the first year following } \\
\text { diagnosis after induction of remission with EEN: } \\
\text { supplemental enteral nutrition is better than nothing! }\end{array}$ & Estudo de Coorte & $\begin{array}{l}\text { BMC } \\
\text { Gastroenterol }\end{array}$ & $\begin{array}{l}\text { Terapia Não } \\
\text { Farmacológica }\end{array}$ & 2014 \\
\hline $\begin{array}{l}\text { Adalimumab and infliximab are equally effective for } \\
\text { Crohn's disease in patients not previously treated with } \\
\text { anti-tumor necrosis factor- } \alpha \text { agents. }\end{array}$ & Estudo de Coorte & $\begin{array}{l}\text { Clin } \\
\text { Gastroenterol } \\
\text { Hepatol } \\
\end{array}$ & $\begin{array}{l}\text { Terapia } \\
\text { Farmacológica }\end{array}$ & 2013 \\
\hline $\begin{array}{l}\text { Adalimumab dose escalation is effective for managing } \\
\text { secondary loss of response in Crohn's disease. }\end{array}$ & Estudo de Coorte & $\begin{array}{l}\text { Aliment } \\
\text { Pharmacol Ther }\end{array}$ & $\begin{array}{l}\text { Terapia } \\
\text { Farmacológica }\end{array}$ & 2014 \\
\hline $\begin{array}{l}\text { Adalimumab-induced interstitial pneumonia in a patient } \\
\text { with Crohn's disease. }\end{array}$ & Relato de Caso & $\begin{array}{l}\text { World J } \\
\text { Gastroenterol }\end{array}$ & $\begin{array}{l}\text { Terapia } \\
\text { Farmacológica }\end{array}$ & 2015 \\
\hline $\begin{array}{l}\text { Adjuvant use of antibiotics with corticosteroids in } \\
\text { inflammatory bowel disease exacerbations requiring } \\
\text { hospitalisation: a retrospective cohort study and meta- } \\
\text { analysis. }\end{array}$ & Estudo de Coorte & $\begin{array}{l}\text { Aliment } \\
\text { Pharmacol }\end{array}$ & $\begin{array}{l}\text { Terapia } \\
\text { Farmacológica }\end{array}$ & 2016 \\
\hline $\begin{array}{l}\text { Anti-TNF } \alpha \text { therapy early improves hemodynamics in } \\
\text { local intestinal and extraintestinal circulations in active } \\
\text { Crohn's disease. }\end{array}$ & Estudo de Coorte & J Crohns Colitis & $\begin{array}{l}\text { Terapia } \\
\text { Farmacológica }\end{array}$ & 2013 \\
\hline $\begin{array}{l}\text { Anti-TNF therapy induced immune neutropenia in } \\
\text { Crohns disease- report of } 2 \text { cases and review of literature. }\end{array}$ & $\begin{array}{l}\text { Relato de Caso } \\
\text { e Revisão de } \\
\text { Literatura }\end{array}$ & J Crohns Colitis & $\begin{array}{l}\text { Terapia } \\
\text { Farmacológica }\end{array}$ & 2012 \\
\hline $\begin{array}{l}\text { Changes in inflammation and quality of life after a single } \\
\text { dose of infliximab during on-going treatment: Differences } \\
\text { between patients with and without IBD symptoms at time } \\
\text { of administration. }\end{array}$ & Estudo de Coorte & $\begin{array}{l}\text { J Pediatr } \\
\text { Gastroenterol } \\
\text { Nutr }\end{array}$ & $\begin{array}{l}\text { Terapia } \\
\text { Farmacológica }\end{array}$ & 2012 \\
\hline $\begin{array}{l}\text { Changes of faecal microbiota in patients with Crohn's } \\
\text { disease treated with an elemental diet and total parenteral } \\
\text { nutrition. }\end{array}$ & Estudo de Coorte & Dig Liver Dis & $\begin{array}{l}\text { Terapia Não } \\
\text { Farmacológica }\end{array}$ & 2012 \\
\hline $\begin{array}{l}\text { Clinical experience with infliximab and adalimumab in } \\
\text { a single-center cohort of patients with Crohn's disease. }\end{array}$ & Estudo de Coorte & $\begin{array}{l}\text { Scand J } \\
\text { Gastroenterol }\end{array}$ & $\begin{array}{l}\text { Terapia } \\
\text { Farmacológica }\end{array}$ & 2012 \\
\hline $\begin{array}{l}\text { Comparative Effectiveness of Infliximab and Adalimumab } \\
\text { for Crohn's Disease. }\end{array}$ & Estudo de Coorte & $\begin{array}{l}\text { Clin } \\
\text { Gastroenterol } \\
\text { Hepatol } \\
\end{array}$ & $\begin{array}{l}\text { Terapia } \\
\text { Farmacológica }\end{array}$ & 2014 \\
\hline $\begin{array}{l}\text { Correlations between skin lesions induced by anti-tumor } \\
\text { necrosis factor- } \alpha \text { and selected cytokines in Crohn's } \\
\text { disease patients. }\end{array}$ & Estudo de Coorte & $\begin{array}{l}\text { World J } \\
\text { Gastroenterol }\end{array}$ & $\begin{array}{l}\text { Terapia } \\
\text { Farmacológica }\end{array}$ & 2014 \\
\hline $\begin{array}{l}\text { Does active Crohn's disease have decreased intestinal } \\
\text { antioxidant capacity? }\end{array}$ & Estudo de Coorte & J Crohns Colitis & $\begin{array}{l}\text { Terapia Não } \\
\text { Farmacológica }\end{array}$ & 2013 \\
\hline $\begin{array}{l}\text { Effect of exclusive enteral nutrition on gut microflora } \\
\text { function in children with Crohn's disease. }\end{array}$ & Estudo de Coorte & $\begin{array}{l}\text { Scand J } \\
\text { Gastroenterol }\end{array}$ & $\begin{array}{l}\text { Terapia Não } \\
\text { Farmacológica }\end{array}$ & 2012 \\
\hline $\begin{array}{l}\text { Effectiveness of infliximab after adalimumab failure in } \\
\text { Crohn's disease. }\end{array}$ & Estudo de Coorte & $\begin{array}{l}\text { World J } \\
\text { Gastroenterol } \\
\end{array}$ & $\begin{array}{l}\text { Terapia } \\
\text { Farmacológica }\end{array}$ & 2012 \\
\hline $\begin{array}{l}\text { Efficacy and tolerability of methotrexate therapy } \\
\text { for refractory Crohn's disease: a large single-centre } \\
\text { experience. }\end{array}$ & Estudo de Coorte & $\begin{array}{l}\text { Aliment } \\
\text { Pharmacol Ther }\end{array}$ & $\begin{array}{l}\text { Terapia } \\
\text { Farmacológica }\end{array}$ & 2012 \\
\hline $\begin{array}{l}\text { Efficacy of Early Immunomodulator Therapy on the } \\
\text { Outcomes of Crohn's Disease. }\end{array}$ & Estudo de Coorte & $\begin{array}{l}\text { BMC } \\
\text { Gastroenterol }\end{array}$ & $\begin{array}{l}\text { Terapia } \\
\text { Farmacológica }\end{array}$ & 2014 \\
\hline
\end{tabular}

Continua. 
Conclusão.

Quadro 1. Descrição dos estudos incluídos na revisão integrativa, segundo título, tipo de estudo, periódicos, enfoque e ano de publicação.

\begin{tabular}{|c|c|c|c|c|}
\hline Título & Tipo de estudo & Periódico & Enfoque & Ano \\
\hline $\begin{array}{l}\text { Fecal microbiota transplantation for severe enterocolonic } \\
\text { fistulizing Crohn's disease. }\end{array}$ & Relato de Caso & \begin{tabular}{|l|} 
World J \\
Gastroenterol
\end{tabular} & $\begin{array}{l}\text { Terapia Não } \\
\text { Farmacológica }\end{array}$ & 2013 \\
\hline $\begin{array}{l}\text { Life-threatening disseminated tuberculosis as a } \\
\text { complication of treatment by infliximab for Crohn's } \\
\text { disease: report of two cases, including cerebral } \\
\text { tuberculomas and miliary tuberculosis. }\end{array}$ & Relato de Caso & J Crohns Colitis & $\begin{array}{l}\text { Terapia } \\
\text { Farmacológica }\end{array}$ & 2012 \\
\hline $\begin{array}{l}\text { Lymphomatoid granulomatosis associated with } \\
\text { azathioprine therapy in Crohn disease. }\end{array}$ & Relato de Caso & $\begin{array}{l}\text { BMC } \\
\text { Gastroenterol }\end{array}$ & $\begin{array}{l}\text { Terapia } \\
\text { Farmacológica }\end{array}$ & 2014 \\
\hline $\begin{array}{l}\text { Mercaptopurine-induced hepatoportal sclerosis in a } \\
\text { patient with Crohn's disease. }\end{array}$ & Relato de Caso & J Crohns Colitis & $\begin{array}{l}\text { Terapia } \\
\text { Farmacológica }\end{array}$ & 2013 \\
\hline $\begin{array}{l}\text { Pneumocystosis in a patient with Crohn's disease treated } \\
\text { with combination therapy with adalimumab. }\end{array}$ & Relato de Caso & J Crohns Colitis & $\begin{array}{l}\text { Terapia } \\
\text { Farmacológica }\end{array}$ & 2012 \\
\hline $\begin{array}{l}\text { Pre-clinical Crohn's disease: diagnosis, treatment and six } \\
\text { year follow-up. }\end{array}$ & Relato de Caso & J Crohns Colitis & $\begin{array}{l}\text { Terapia } \\
\text { Farmacológica }\end{array}$ & 2014 \\
\hline $\begin{array}{l}\text { Predictors of dose escalation of adalimumab in a } \\
\text { prospective cohort of Crohn's disease patients. }\end{array}$ & Estudo de Coorte & $\begin{array}{l}\text { Aliment } \\
\text { Pharmacol Ther }\end{array}$ & $\begin{array}{l}\text { Terapia } \\
\text { Farmacológica }\end{array}$ & 2012 \\
\hline $\begin{array}{l}\text { Prospective Evaluation of the Achievement of Mucosal } \\
\text { Healing with Anti-TNF- } \alpha \text { Therapy in a Paediatric Crohn's } \\
\text { Disease Cohort. }\end{array}$ & Estudo de Coorte & J Crohns Colitis & $\begin{array}{l}\text { Terapia } \\
\text { Farmacológica }\end{array}$ & 2016 \\
\hline $\begin{array}{l}\text { Reversible Henoch-Schönlein purpura complicating } \\
\text { adalimumab therapy. }\end{array}$ & Relato de Caso & J Crohns Colitis & $\begin{array}{l}\text { Terapia } \\
\text { Farmacológica }\end{array}$ & 2012 \\
\hline $\begin{array}{l}\text { Severe adalimumab-induced thrombocytopenia in a } \\
\text { patient with Crohn's disease. }\end{array}$ & Relato de Caso & J Crohns Colitis & $\begin{array}{l}\text { Terapia } \\
\text { Farmacológica }\end{array}$ & 2012 \\
\hline $\begin{array}{l}\text { Smoking does influence disease behaviour and impacts } \\
\text { the need for therapy in Crohn's disease in the biologic } \\
\text { era. }\end{array}$ & Estudo de Coorte & $\begin{array}{l}\text { Aliment } \\
\text { Pharmacol Ther }\end{array}$ & $\begin{array}{l}\text { Terapia } \\
\text { Farmacológica }\end{array}$ & 2013 \\
\hline $\begin{array}{l}\text { Successful Mercaptopurine Usage despite Azathioprine- } \\
\text { Induced Pancreatitis in Paediatric Crohn's Disease. }\end{array}$ & Relato de Caso & J Crohns Colitis & $\begin{array}{l}\text { Terapia } \\
\text { Farmacológica }\end{array}$ & 2015 \\
\hline $\begin{array}{l}\text { Surgery and postoperative recurrence in children with } \\
\text { Crohn disease. }\end{array}$ & Estudo de Coorte & $\begin{array}{l}\text { J Pediatr } \\
\text { Gastroenterol } \\
\text { Nutr }\end{array}$ & $\begin{array}{l}\text { Terapia Não } \\
\text { Farmacológica }\end{array}$ & 2015 \\
\hline $\begin{array}{l}\text { The risks of post-operative complications following } \\
\text { pre-operative infliximab therapy for Crohn's disease in } \\
\text { patients undergoing abdominal surgery: a systematic } \\
\text { review and meta-analysis. }\end{array}$ & $\begin{array}{l}\text { Revisão } \\
\text { Sistemática e } \\
\text { Metanalise }\end{array}$ & J Crohns Colitis & $\begin{array}{l}\text { Terapia } \\
\text { Farmacológica }\end{array}$ & 2013 \\
\hline
\end{tabular}

\section{DISCUSSÃO}

\section{Terapia farmacológica}

Os pacientes com doença de Crohn necessitarão de medicamentos anti-inflamatórios durante décadas. O seu tratamento é baseado em esteroides e imunossupressores, mas em pacientes não responsivos, em compostos biológicos, como os anti-TNF- $\alpha$ e anticorpos. ${ }^{10,11}$

Os medicamentos anti-TNF são usados para indução e manutenção da remissão em pacientes com DC. ${ }^{12} \mathrm{Na}$ doença de Crohn ativa, a primeira administração de anti-TNF rapidamente normaliza os marcadores inflamatórios do plasma e o fluxo sanguíneo nas artérias mesentéricas e retrobulbares, concomitantemente, sem afetar a pressão arterial e função endotelial. ${ }^{13}$

As lesões cutâneas em pacientes com DC, durante a terapia biológica, podem resultar, de forma significativa, no aumento das concentrações de algumas interleucinas (IL), como IL17A e IL-23, que são fortemente associados com as vias imunitárias TNF- $\alpha /$ Th1. ${ }^{14}$

Além disso, essas drogas apresentam diversos efeitos secundários, que são causados por um decréscimo na resposta inflamatória, o que leva ao aumento da susceptibilidade a infecções oportunistas, ${ }^{10,15}$ tais como infecções por fungos, ${ }^{10}$ especialmente a reativação com Mycobacterium tuberculosis. ${ }^{15,16}$ Devido a isto, o rastreio da tuberculose e, eventualmente, 
quimioterapia preventiva, deve tornar-se o padrão de cuidado para indivíduos submetidos a terapias $\alpha$-antagonistas. ${ }^{16}$

As infecções por micobactérias atípicas, tais como Mycobacterium marinum, são raras, ${ }^{15}$ bem como a trombocitopenia. ${ }^{12}$ A infecção por $M$. marinum tende a ter um curso difícil e prolongado, devido ao diagnóstico tardio, sendo indicada a descontinuação da terapia com anti-TNF. ${ }^{15}$ Vale salientar que os agentes anti-TNF podem produzir agranulocitose auto-imune, pois eles podem desencadear a produção de anticorpos de granulócitos. ${ }^{17}$

Há vários relatos de doença pulmonar induzida por anti fator de necrose tumoral, especialmente em pacientes com doenças reumatológicas. Embora os distúrbios pulmonares possam ser uma manifestação extra-intestinal da doença inflamatória do intestino, a terapia biológica também pode ser uma causa da lesão pulmonar. ${ }^{1}$

A lesão pulmonar secundária à terapia anti-TNF deve, após a exclusão de outras etiologias, ser considerada em pacientes que têm uma associação temporal entre o início dos sintomas respiratórios e a exposição a estas drogas. Um padrão compatível na biópsia e a melhora clínica após a descontinuação da droga anti-TNF apoiam fortemente o diagnóstico. ${ }^{1}$ Ademais, foi visto que os fumantes com doença de Crohn possuem um curso mais grave da doença, com aumento das necessidades terapêuticas, quando comparado com os não-fumantes. ${ }^{18}$

Os anticorpos contra o fator de necrose tumoral- $\alpha$ são amplamente utilizados no tratamento da doença de Crohn. O infliximabe (IFX) e adalimumabe (ADA) são os medicamentos anti-TNF mais comumente utilizados para DC. Foi observada eficácia semelhante de infliximabe e adalimumabe para essa doença. ${ }^{19}$

O ADA é uma droga anti-TNF utilizada para induzir e manter a remissão em pacientes com doenças mediadas pelo sistema imunológico, tais como a doença de Crohn. ${ }^{1}$

A eficácia do adalimumabe na manutenção da remissão em pacientes com doença de Crohn pode diminuir ao longo do tempo, levando à perda secundária de resposta, a qual muitas vezes é conduzida com o aumento da dose..$^{20,21}$ Esse ajuste da dose é eficaz para recapturar a resposta sintomática após a perda secundária de resposta, porém, mais da metade desses pacientes acabará tendo uma perda terciária de resposta. ${ }^{20}$ Ademais, índice de massa corporal (IMC) alto e falha na resposta secundária ao tratamento com IFX são fatores preditivos para um aumento da dose durante o tratamento com adalimumabe. ${ }^{21}$

A mudança do ADA para IFX pode ser útil em pacientes que desenvolvem efeitos adversos ou perda de resposta. No entanto, o benefício do infliximabe em não responsivos primários não foi estabelecido. ${ }^{22}$

O IFX é usado cada vez mais como terapia de manutenção das DII. ${ }^{23}$ As concentrações do fármaco abaixo de $3 \mathrm{ng} / \mathrm{mL}$ podem aumentar a probabilidade de sintomas e inflamação. ${ }^{24}$
Uma única dose de infliximabe resulta na diminuição da proteína $\mathrm{C}$ reativa $(\mathrm{PCR})$ de alta sensibilidade, uma melhoria que é particularmente conhecida entre os indivíduos que são sintomáticos no momento do tratamento. Embora sejam necessários estudos randomizados, estes dados observacionais podem auxiliar os médicos, pacientes e famílias a respeito das expectativas sobre a época e a extensão dessas mudanças após dose única desse medicamento. ${ }^{23}$

Segundo Rosenfeld, Qian e Bressler (2013), o IFX pode ser seguro para ser utilizado no pré-operatório, sem aumentar o risco de complicações pós-operatórias para pacientes com doença de Crohn submetidos à cirurgia abdominal. ${ }^{25}$ Marques et al (2012) ressaltam que o infliximabe pode ser usado com segurança em doentes com Púrpura de Henoch-Schönlein (PHS) relacionada ao ADA. ${ }^{26}$

$\mathrm{O}$ adalimumabe, embora seja teoricamente menos relacionado às reações imunomediadas, ainda pode apresentar efeitos adversos que devem ser observados pelos médicos. ${ }^{26}$ Poucos casos de toxicidade pulmonar induzida por ADA têm sido relatados e a maioria deles são em pacientes com doenças reumatológicas. ${ }^{1}$

A eficácia de IFX e ADA parece aumentar quando administrados com terapia imunomoduladora, embora seja de forma mais significativa no IFX. ${ }^{27}$ Nuti et al (2016) mostraram que a terapia biológica com infliximabe e adalimumabe é eficaz no alcance da manutenção da cicatrização da mucosa, melhorando acentuadamente lesões da mucosa em pacientes com DC pediátricos. ${ }^{28}$ Sorrentino et al (2014) concluíram que o infliximabe pareceu capaz de induzir essa cura da mucosa, no entanto, a DC reapareceu meses após a medicação ser cessada. ${ }^{29}$

Em um estudo de coorte, percebeu-se que a combinação de produtos biológicos e imunomoduladores foi mais eficaz do que a monoterapia biológica na melhoria das lesões nas mucosas, porém, a terapia de combinação não influenciou significativamente no resultado dos doentes e na duração da doença. ${ }^{28}$

Kwak et al (2014) afirmam que a terapêutica imunomoduladora inicial é mais eficaz do que a terapia convencional em induzir a remissão, mas não na prevenção da recaída. Um importante exame utilizado é a PCR, sendo um indicador significativo de recaída. ${ }^{30}$

Já o metotrexato, segundo Suares et al (2012), é eficaz na indução da remissão e prevenção da recaída da DC como um imunossupressor de primeira linha, porém os dados de eficácia após a falha ou intolerância a tiopurinas são limitados. Logo, o metotrexato é eficaz, em termos de resposta inicial, nos pacientes com doença de Crohn que falharam ou são intolerantes a tiopurinas, no entanto, sua eficácia não é sustentada a longo prazo. ${ }^{31}$

As tiopurinas desempenham um papel central na gestão da doença inflamatória do intestino, ${ }^{32}$ sendo utilizada na terapia a longo prazo. ${ }^{33}$ A azatioprina (AZA) e mercaptopurina (MP) são recomendados para a manutenção da remissão livre de esteroides em crianças com doença de Crohn. ${ }^{34}$ 
A AZA e a MP têm sido associadas a um número de anormalidades do fígado, incluindo a hepatite, doença venooclusiva, hiperplasia nodular regenerativa e peliose hepática. Assim, deve ser ressaltada a importância da monitorização da função hepática nos pacientes que fazem uso dessas drogas. ${ }^{32}$

Os acontecimentos hepáticos adversos graves podem surgir silenciosamente e passar despercebidos durante o tratamento com MP e, devido a isso, faz-se necessária a interrupção desse medicamento nesses casos. ${ }^{32}$

Além disso, a terapia com tiopurina também está relacionada com um aumento da incidência de doenças linfoproliferativas. A terapia de longo prazo com tiopurina continua sendo útil para a gestão de DII, sendo essencial que os pacientes e médicos estejam cientes de todos os seus efeitos adversos. ${ }^{33}$

A pancreatite induzida por AZAé um efeito colateral importante e tem sido considerada como uma contraindicação absoluta para a utilização de uma segunda tiopurina em pacientes com DII. ${ }^{34}$ Porém, segundo Gallego-Gutiérrez et al (2015), esse evento adverso não deve ser considerado como uma contraindicação absoluta para o uso de MP. Uma investigação mais aprofundada é necessária para possibilitar uma melhor compreensão dos mecanismos subjacentes aos eventos adversos, o que pode permitir mais possibilidades terapêuticas.

A adição de antibióticos a esteroides intravenosos, para o tratamento das exacerbações das DII, foi associada a uma menor necessidade de terapia de resgate médico-hospitalar em colite ulcerativa, sem benefício significativo a longo prazo, não interferindo nos resultados a curto ou longo prazo na doença de Crohn. ${ }^{4}$

\section{Terapia não farmacológica}

Além do tratamento medicamentoso, também foi relatada a importância da utilização da nutrição parenteral total (NPT) e da nutrição enteral exclusiva (NEE). A NPT mostrouse importante na melhoria do estado nutricional, além de diminuir a atividade inflamatória. Sua administração é recomendada para pacientes com DC moderada a grave até a remissão clínica, a fim de minimizar o risco de complicações pós-operatórias precoces. ${ }^{35}$

Duncan et al (2014) relataram que algumas pesquisas sugerem que a nutrição enteral de manutenção contínua pode ser benéfica na manutenção da remissão da doença de Crohn. ${ }^{36} \mathrm{~A}$ NEE causa impactos na composição da microbiota intestinal e alterações na atividade metabólica fecal, ${ }^{37}$ sendo um tratamento de primeira linha em crianças com DC ativa, mas é raramente usada em adultos com a doença ativa. ${ }^{38}$

Segundo Tjellström et al (2012), o efeito anti-inflamatório da NEE em crianças com DC ativa em intestino delgado/colon é provocada por alterações da atividade da microflora intestinal. Por outro lado, ele evidenciou que, em seu estudo, nenhuma das crianças com doença perianal apresentou melhora clínica ou bioquímica após o tratamento com NEE. ${ }^{38}$

Um estudo feito por Hansen et al (2015) revelou uma alta taxa de recorrência de DC após a primeira ressecção cirúrgica, além de uma necessidade frequente de reoperação e uma necessidade decrescente de cirurgia no período após a introdução de imunomoduladores. Ademais, foi demonstrada uma diminuição nas taxas de cirurgia primária após a introdução de AZA, porém, não foi encontrada nenhuma diferença nas taxas de cirurgia ao se comparar pacientes tratados com e sem azatioprina. Isso reflete em um aumento geral da qualidade do tratamento, levando a uma menor necessidade de ressecção intestinal. Além disso, o tratamento pós-operatório com AZA não modificou a taxa de recorrência e o grupo de pacientes que recebeu essa droga teve o curso da doença no pré-operatório mais grave do que o grupo que não recebeu qualquer tratamento no pós-operatório. ${ }^{6}$

Zhang et al (2013) propuseram que o transplante de microbiota fecal (TMF) possa ser uma terapia de resgate promissora para a DII refratária. Ademais, ainda relatou o sucesso do tratamento com TMF padronizado em um caso de DC refratária complicada com fístula, sulfato de bário residual e formação de grande massa inflamatória intraperitoneal, sendo o primeiro caso de DC grave tratado com uso de TMF. ${ }^{39}$

\section{CONCLUSÕES}

Dentre os estudos analisados, $81,8 \%$ foram relacionados à terapia farmacológica da Doença de Crohn e, acerca dessa terapia, houve um maior enfoque no uso de anti-TNF e o seu uso combinado com imunomoduladores em casos mais graves, observando-se uma necessidade menor de cirurgia naqueles que fizeram uso de adalimumabe. As tiopurinas também foram mencionadas principalmente para a manutenção livre de esteroides, em especial em crianças, a despeito de seus diversos efeitos colaterais.

A respeito da terapia não farmacológica, a NPT foi mencionada para o uso em pacientes com DC moderada a grave até a remissão clínica. Já a NEE foi identificada como tratamento de primeira linha em crianças com DC ativa e raramente utilizada em adultos. O uso de TMF foi proposto como uma terapia de resgate alternativa em casos de DC refratária complicada.

Comparando essas duas terapêuticas, observou-se que há uma prevalência de estudos a respeito das terapias farmacológicas quando comparadas com as não farmacológicas (18,2\%), sendo que a última atualmente ainda é pouco explorada.

Ademais, constatou-se uma escassez de artigos acerca desse tema nos anos mais recentes, visto que a maioria dos encontrados foram do ano de 2012 (36,4\%). Também percebeu-se uma maior frequência de estudos de coorte e relatos de caso durante esses anos, que, juntos, compreenderam 90,9\% dos artigos analisados.

Assim, vale ressaltar que atualmente ainda há necessidade de realização de novos estudos sobre as opções terapêuticas da $\mathrm{DC}$, principalmente no que diz respeito à terapia não farmacológica, visando uma menor taxa de complicações, recorrência e de efeitos colaterais desencadeadas por essas drogas, a fim de que seja garantido um tratamento eficaz e seguro para os pacientes acometidos por essa doença. 


\section{REFERÊNCIAS}

1. Casanova MJ, Chaparro M, Valenzuela C, Cisneros C, Gisbert JP. Adalimumab-induced interstitial pneumonia in a patient with Crohn's disease. World J Gastroenterol. 2015;21(7):2260-2.

2. Beser OF, Conde CD, Serwas NK, Cokugras FC, Kutlu T, Boztug $\mathrm{K}$, et al. Clinical features of interleukin 10 receptor gene mutations in children with very early-onset inflammatory bowel disease. J Pediatr Gastroenterol Nutr. 2015;60(3):332-8.

3. Boland BS, Boyle DL, Sandborn WJ, Firestein GS, Levesque BG, Hillman J, et al. Validation of gene expression biomarker analysis for biopsy-based clinical trials in Crohn's disease. Inflamm Bowel Dis. 2015;21(2):323-30

4. Gupta V, Rodrigues R, Nguyen D, Sauk J, Khalili H, Yajnik V, et al. Adjuvant use of antibiotics with corticosteroids in inflammatory bowel disease exacerbations requiring hospitalisation: a retrospective cohort study and meta-analysis. Aliment Pharmacol Ther. 2016;43(1):52-60.

5. Biancheri P, Brezski RJ, Di Sabatino A, Greenplate AR, Soring KL, Corazza GR, et al. Proteolytic cleavage and loss of function of biologic agents that neutralize tumor necrosis factor in the mucosa of patients with inflammatory bowel disease. Gastroenterology. 2015;149(6):1564-1574.e3.

6. Hansen LF, Jakobsen C, Paerregaard A, Qvist N, Wewer V. Surgery and postoperative recurrence in children with Crohn disease. J Pediatr Gastroenterol Nutr. 2015;60(3):347-51 .

7. Benefield LE. Implementing evidence-based practice in home care. Home Healthc Nurse. 2003;21(12):804-11.

8. Polit DF, Beck CT. Using research in evidence-based nursing practice. In: Polit DF, Beck CT, editors. Essentials of nursing research. Methods, appraisal and utilization. Philadelphia (USA): Lippincott Williams \& Wilkins; 2006. p. 457-94.

9. Souza MT, Silva MD, Carvalho R. Revisão integrativa: o que é e como fazer. Einstein. 2010;8(1 Pt 1):102-6.

10. Desales AL, Mendez-Navarro J, Méndez-Tovar LJ, Ortiz-Olvera NX, Cullen G, Ocampo J, et al. Pneumocystosis in a patient with Crohn's disease treated with combination therapy with adalimumab. J Crohns Colitis. 2012;6(4):483-7.

11. Riis A, Martinsen TC, Waldum HL, Fossmark R. Clinical experience with infliximab and adalimumab in a single-center cohort of patients with Crohn's disease. Scand J Gastroenterol. 2012;47(6):649-57.

12. Casanova MJ, Chaparro M, Martínez S, Vicuña I, Gisbert JP. Severe adalimumab-induced thrombocytopenia in a patient with Crohn's disease. J Crohns Colitis. 2012;6(10):1034-7.

13. Bonnin P, Coelho J, Pocard M, Levy BI, Marteau P. Anti$\mathrm{TNF} \alpha$ therapy early improves hemodynamics in local intestinal and extraintestinal circulations in active Crohn's disease. J Crohns Colitis. 2013;7(6):451-9.

14. Włodarczyk M, Sobolewska A, Wójcik B, Loga K, Fichna J, Wiśniewska-Jarosińska M. Correlations between skin lesions induced by anti-tumor necrosis factor- $\alpha$ and selected cytokines in Crohn's disease patients. World J Gastroenterol. 2014;20(22):7019-26.
15. Kump PK, Högenauer C, Wenzl HH, Petritsch W. A case of opportunistic skin infection with Mycobacterium marinum during adalimumab treatment in a patient with Crohn's disease. J Crohns Colitis. 2013;7(1):e15-8.

16. Tissot C, Couraud S, Meng L, Girard P, Avrillon V, Gérinière L, et al. Life-threatening disseminated tuberculosis as a complication of treatment by infliximab for Crohn's disease: report of two cases, including cerebral tuberculomas and miliary tuberculosis. J Crohns Colitis. 2012;6(9):946-9.

17. Sebastian S, Ashton K, Houston Y, Diggory TM, Dore P. AntiTNF therapy induced immune neutropenia in Crohns disease- report of 2 cases and review of literature. J Crohns Colitis. 2012;6(6):713-6.

18. Nunes T, Etchevers MJ, Domènech E, García-Sánchez V, Ber Y, Peñalva M, et al. Smoking does influence disease behaviour and impacts the need for therapy in Crohn's disease in the biologic era. Aliment Pharmacol Ther. 2013;38(7):752-60.

19. Osterman MT, Haynes K, Delzell E, Zhang J, Bewtra M, Brensinger $\mathrm{C}$, et al. Comparative effectiveness of infliximab and adalimumab for Crohn's disease. Clin Gastroenterol Hepatol. 2014;12(5):811-7.

20. Ma C, Huang V, Fedorak DK, Kroeker KI, Dieleman LA, Halloran $\mathrm{BP}$, et al. Adalimumab dose escalation is effective for managing secondary loss of response in Crohn's disease. Aliment Pharmacol Ther. 2014;40(9):1044-55.

21. Bultman E, Haar C, van Liere-Baron A, Verhoog H, West RL, Kuipers EJ, et al. Predictors of dose escalation of adalimumab in a prospective cohort of Crohn's disease patients. Aliment Pharmacol Ther. 2012;35(3):335-41.

22. Chaparro M, Andreu M, Acosta MB, García-Planella E, Ricart $\mathrm{E}$, Domènech $\mathrm{E}$, et al. Effectiveness of infliximab after adalimumab failure in Crohn's disease. World J Gastroenterol. 2012;18(37):521924.

23. DeBoer MD, Barnes BH, Stygles NA, Sutphen JL, Borowitz SM. Changes in inflammation and quality of life after a single dose of infliximab during on-going treatment: Differences between patients with and without IBD symptoms at time of administration. J Pediatr Gastroenterol Nutr. 2012,54(4):486-90.

24. Levesque BG, Greenberg GR, Zou G, Sandborn WJ, Singh S, Hauenstein $S$, et al. A prospective cohort study to determine the relationship between serum infliximab concentration and efficacy in patients with luminal Crohn's disease. Aliment Pharmacol Ther. 2014;39(10):1126-35.

25. Rosenfeld G, Qian H, Bressler B. The risks of post-operative complications following pre-operative infliximab therapy for Crohn's disease in patients undergoing abdominal surgery: a systematic review and meta-analysis. J Crohns Colitis. 2013;7(11):868-77.

26. Marques I, Lagos A, Reis J, Pinto A, Neves B. Reversible Henoch-Schönlein purpura complicating adalimumab therapy. J Crohns Colitis. 2012;6(7):796-9.

27. Kestens C, van Oijen MG, Mulder CL, van Bodegraven AA, Dijkstra G, de Jong D, et al. Adalimumab and infliximab are equally effective for Crohn's disease in patients not previously treated with 
anti-tumor necrosis factor- $\alpha$ agents. Clin Gastroenterol Hepatol. 2013;11(7):826-31.

28. Nuti F, Civitelli F, Bloise S, Oliva S, Aloi M, Latorre G, et al. Prospective evaluation of the achievement of mucosal healing with anti-TNF- $\alpha$ therapy in a paediatric crohn's disease cohort. J Crohns Colitis. 2016;10(1):5-12.

29. Sorrentino D, Avellini C, Geraci M, Vadalà S. Pre-clinical crohn's disease: diagnosis, treatment and six year follow-up. J Crohns Colitis. 2014;8(7):702-7.

30. Kwak MS, Kim DH, Park SJ, Kim TI, Hong SP, Kim WH, et al. Efficacy of early immunomodulator therapy on the outcomes of Crohn's disease. BMC Gastroenterol. 2014;14:85.

31. Suares NC, Hamlin PJ, Greer DP, Warren L, Clark T, Ford AC. Efficacy and tolerability of methotrexate therapy for refractory Crohn's disease: a large single-centre experience. Aliment Pharmacol Ther. 2012;35(2):284-91.

32. Tuyama AC, Krakauer M, Alzaabi M, Fiel MI, Legnani P, Schiano TD. Mercaptopurine-induced hepatoportal sclerosis in a patient with Crohn's disease. J Crohns Colitis. 2013;7(7):590-3.

33. Connors W, Griffiths C, Patel J, Belletrutti PJ. Lymphomatoid granulomatosis associated with azathioprine therapy in Crohn disease. BMC Gastroenterol. 2014;14:127.
34. Gallego-Gutiérrez S, Navas-López VM, Kolorz M, Bartosova L, Lukac K, Luque-Pérez S, et al. Successful mercaptopurine usage despite azathioprine-induced pancreatitis in paediatric crohn's disease. J Crohns Colitis. 2015;9(8):676-9.

35. Pinto MA, Lopes MS, Bastos ST, Reigada CL, Dantas RF, Jaime CB Neto, et al. Does active crohn's disease have decreased intestinal antioxidant capacity? J Crohns Colitis. 2013;7(9):e358-66.

36. Duncan H, Buchanan E, Cardigan T, Garrick V, Curtis L, McGrogan $\mathrm{P}$, et al. A retrospective study showing maintenance treatment options for paediatric $\mathrm{CD}$ in the first year following diagnosis after induction of remission with EEN: supplemental enteral nutrition is better than nothing! BMC Gastroenterol. 2014;14:50.

37. Shiga H, Kajiura T, Shinozaki J, Takagi S, Kinouchi Y, Takahashi $\mathrm{S}$, et al. Changes of faecal microbiota in patients with Crohn's disease treated with an elemental diet and total parenteral nutrition. Dig Liver Dis. 2012;44:736-42.

38. Tjellström B, Högberg L, Stenhammar L, Magnusson KE, Midtvedt T, Norin E, et al. Effect of exclusive enteral nutrition on gut microflora function in children with Crohn's disease. Scand J Gastroenterol. 2012;47(12):1454-9.

39. Zhang FM, Wang HG, Wang M, Cui BT, Fan ZN, Ji GZ. Fecal microbiota transplantation for severe enterocolonic fistulizing Crohn's disease. World J Gastroenterol. 2013;19(41):7213-16.

\section{Como citar:}

Sousa GF, Grochoski KC, Marinho RF, Vieira MF, Nahoum RG. Doença de Crohn: aspectos do tratamento na atualidade. Rev Med UFC. 2019 out-dez;59(4):62-69. 